\title{
Prosumeristic Publications: alt+yd
}

\begin{abstract}
This article describes the design process followed in the making of a prosumerist publication. Alt +yd is a printed zine that goes beyond one-way communication with its readers. It takes into account the reactions of Instagrammers to its digital publication and using a program, incorporates it into its printed self. Inspired from the Open Source and Open Data culture, project alt $+y d$ reflects upon the kind and medium of content most consumed today, and the relevance and possible adaptations of traditional media to keep in pace with emerging digital culture.
\end{abstract}

\section{Keywords}

Prosumerism, Internet Culture, Design Process, Print, Editorial Design

\section{Introduction}

In this article we present to you an experiment in editorial design, the output of which is a zine named alt+yd. The experiment involved integrating social media as a programmed content source for a print publication. Simply put, we created a program that would convert Instagram profile data into printable publication spreads. We believe writing about this editorial design process is of importance as our experiment is potentially one of several precursory examples exploring the future of print publication.

\section{Background}

The National Institute of Design is India's premier design education, service, and research institution. Every year at the school, hundreds of students sit for the annual campus recruitment event, colloquially called the 'placements'. There are two kinds of opportunities at the event: for students seeking graduation thesis internships and for graduates seeking full-time jobs. Those who fit the cookie-cutter requirements of the industry are selected and often offered large starting packages.

The 'placements' is a good platform to meet potential employers if what the student seeks is to work in these said companies. However, most students are in the process of figuring out what they want to do and find themselves to not fit the demands of the campus recruitment companies. Students whose portfolios do not adhere to the traditional interpretations of their disciplines find it difficult to market their design approach and practice. Furthermore, younger design students inadvertently begin looking up to the industry norms thereby preventing themselves from exploring the field independently. 
In light of these micro-conflicts, discussions arose and a shared desire was expressed by the authors to create a platform that showcases alternative projects and celebrates experimentation. The aim was to highlight projects that touch upon non-mainstream subjects and methodologies but often go unnoticed. Therein the idea of a publication documenting such projects emerged. The name alt+yd is a take on the Young Designer (YD) book, a compilation of all the graduation projects, published every year by the National Institute of Design (NID).

\section{Intent of the Zine}

Alt $+y d$ is a curation of graduation thesis projects done at NID that explore emerging design practices and futures of the country. By showcasing alternative possibilities of graduation projects, alt+yd aims to be a source of inspiration for budding design students and is intended to be a statement to the design industry on the fresh possibilities that the field provides.

\section{An Experiment in Editorial Design}

Publication has traditionally been a one-way communication platform where the printed material communicates to the reader. The authors decided to ask- what might a book look like if every reader, in some way, could contribute to the published content? In the ever-connected world of the internet, how might one achieve that?

Fig. 1. (left to right) The Instagram post, the program, the generated spread, the printed book

\section{Prosumerism}

A Prosumer[3] is a person who produces as well as consumes a product. In the context of the early $21 \mathrm{st}$ century, the term 'Prosumerism' blurs the meaning of the term 'product' itself. On almost all digital platforms today, the consumer of the digital content is often also the producer of the said content. A give and take of data to and from the people runs most systems today. Interesting results can be seen if the concept of prosumerism is applied to other, traditionally one-way media.

\section{Prosumeristic Publication: A Note on Production Methodology}

What might we get if we 'Ctrl+P' an Instagram profile? Given its wide reach and ease of use, Instagram was chosen as the social media platform to display alt+yd. An Instagram page (@altplusyd) was created for the project. The primary content of the zine- a curated collection of provocative projects was regularly updated on the page. The aim of the page was to elicit dialogue and reactions to the digitally published projects.

An application was custom developed in the Processing Development Environment (PDE)[4] to extract all the content of the Instagram page and convert it into print ready spreads. This application exploited Instagram data available to its users in the form of JSON[5] files. These files helped with the extraction of two kinds of data- 1) The metadata: the project title and the department was extracted from the hashtags, 2) Project information: the project description, comments and likes were pulled from the post. The consequent spreads therefore contained not only the projects but also the reactions and comments they had garnered on Instagram,
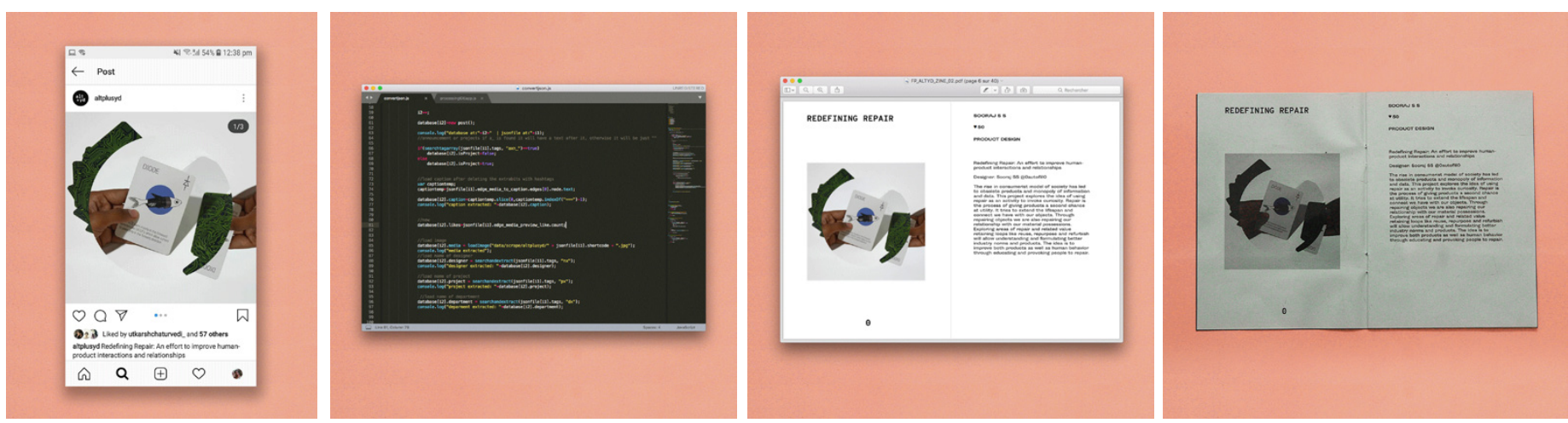
ensuring that the same audience who would read the book in the future could contribute to its contents in the present.

The additional standard pages of a publication such as the title, conclusion and print notes were created in InDesign and added to the generated PDF of the main text block. The technique of foiling was used for the cover page, each cover was foiled by hand, giving each copy a unique appearance. The cover and the text block were then center-stapled and cut.

The first edition of alt $+y d$ was available at the 39th Convocation Ceremony of the National Institute of Design, 2019. Every printed edition thereafter will be different from its predecessor due to its ever evolving content.

\section{Freedoms and Constraints of the Project}

The authors had total freedom on the design and production of the project. Constraints were few but hefty. The project was conceived on December 1 , 2018 and had to be delivered by January 10, 2019. The entire timeline for conceptualization, prototyping, and production was all within a month. The budget of the project was restricted to 5000 INR, i.e. around 70.44 USD.

\section{Reflections}

The eye is well acquainted to how a book reads. So much so that the mind forgets that the book is the way it is, owing to the freedoms and constraints of design and production dating back to the era of the printing press.

A prosumeristic publication comes with its own quirks on how it reads. These quirky elements are not entirely new. It was observed that they merely seemed misplaced to the eye in the context of a printed booklet. The following are the observations and feedback of the readers of alt+yd as well as the authors:

1. The emoji ' $\checkmark 45$ ' (heart icon + a number) is a familiar symbol. Most are aware of what it signifies in the digital world- that 45 people have 'liked' the post. Yet, finding it on printed paper was a new experience.
Fig. 2. Production

cycle of alt+yd
2. Proper nouns were no longer just words. Any name which had an Instagram profile to it was tagged with an '@'. This is a common practice in the digital world, yet, seemed uncommon when every name was followed by the Instagram counterpart of it.

\section{Comments on the digital platform are another} regularly occurring phenomenon. Yet, printed comments seem unusual, even more so when on reading them one realizes that they could be written by bots!

4. The usage of '\#hashtags' to control the content flow from the Instagram posts to the publication- Since not all posts could be a part of the publication due to certain editorial decisions and constraints, a new system was devised to control this content flow using hashtags. These hashtags helped the custom-developed program

Fig. 3. Printed copy of alt+yd, first edition (2019)
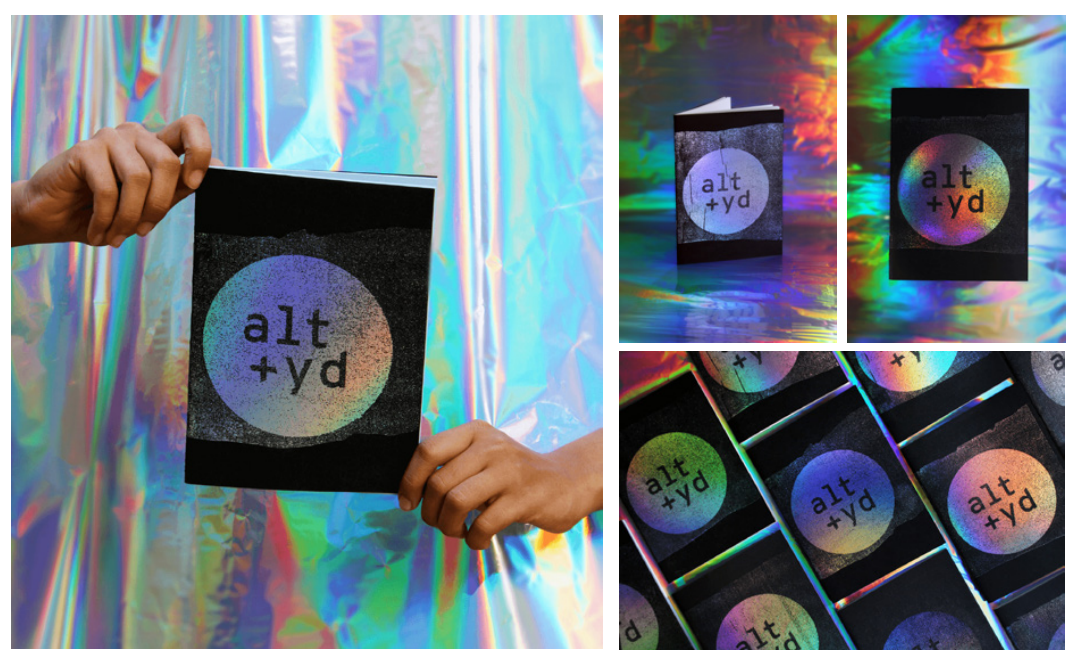
to identify the posts that were to be fully incorporated in the publication, the posts to be included partially as well as those that were supposed to be excluded completely.

\section{Comments that tagged other people, thanked} someone or were emotionally charged, suddenly seemed to have relatively more gravitas in a printed publication than online. An indication of how we still take printed content more seriously than digital?

\section{Conclusion}

This zine explores a future of print publication where the boundary between the digital and the physical medium is blurred. The physical is not merely a tangible copy of the digital. Rather, the physical could borrow from the evolving reactions to the digital, making 'reactions' another form of content. With a futureoriented outlook, the approach with which alt+yd was made asks the following questions:

'In what ways might the boundary between the digital and the printed be blurred?'

'How might 'open source' be interpreted in the world of print publication?'

'Can a reader contribute to a book even after it has been printed?'

Fig. 4. Example of a printed comment, potentially from a bot account, alt+yd (January, 2019)

\section{References}

1. Howe, Jeff (June 2, 2006). "Crowdsourcing: A Definition". Crowdsourcing Blog. Retrieved January 2, 2013.

2. Toffler, Alvin. The Third Wave. Bantam Books, 1990.

3. Ayres, Carly. Crowdsourced Résumé, http:// www.carlyayres.com/

4. Processing Development Environment (PDE), https:// processing.org/reference/environment/\#PDE

5. JavaScript Object Notation, https://www.json.org/

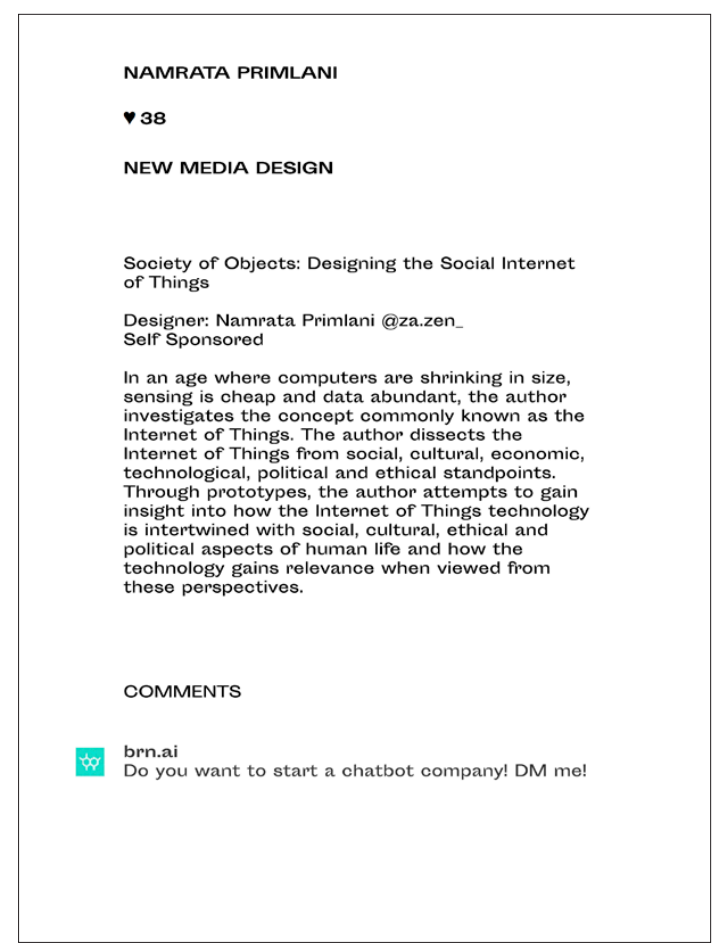

